\title{
A review of the applications for involuntary admissions made to the Mental Health Review Boards by institutions in Gauteng in 2008
}

M Y H Moosa, MMed Psych, FC Psych, MCFP

F Y Jeenah, MMed Psych, FC Psych

Division of Psychiatry, University of the Witwatersrand, Johannesburg

Aim. To review applications for involuntary admissions made to the Mental Health Review Boards (MHRBs) by institutions in Gauteng.

Method. A retrospective review of the register/database of the two review boards in Gauteng for the period January December 2008. All applications for admissions (involuntary and assisted inpatient) and outpatient care (involuntary and assisted), and periodic reports for continued care (inpatient or outpatient care) were included.

Results. During the study period the two MHRBs received a total of 3803 applications for inpatient care, of which 2526 were for assisted inpatient care $148.1 \%$ regional hospitals, $29.6 \%$ specialised psychiatric hospitals, $22.2 \%$ tertiary academic hospitals). Of the applications for involuntary inpatient care, $73.1 \%$ were from the specialised psychiatric hospitals $165.2 \%$ from Sterkfontein Hospital). Applications for outpatient care, treatment and rehabilitation (CTR) numbered 1 226 (92\% assisted outpatient CTR). Although the health establishments in northern Gauteng applied for more outpatient CTR compared with those in southern Gauteng (879 v. 347, respectively), the ratios of assisted to involuntary outpatient applications for CTR for each region were similar (approximately 12:1 and 9:1, respectively). The boards received 3805 periodic reports for prolonged CTR (93.5\% inpatient, $6.5 \%$ outpatient) in the majority of cases for assisted CTR.

Conclusion. The study suggests that in the 4 years since the promulgation of the Mental Health Care Act (MHCA) in 2004, there have been significant strides towards implementation of the procedures relating to involuntary admission and CTR by all stakeholders. Differences in levels of implementation by the various stakeholders may result from differences in knowledge, perceptions, attitudes and understanding of their roles and therefore indicate the need for education of mental health care professionals and the public on a massive scale. The Department of Health also needs to invest more funds to improve mental health human resources and infrastructure at all health establishments.

The Mental Health Care Act No. 17 of 2002 (MHCA)' replaced the Mental Health Act No. 18 of 1973 in South Africa in December 2004. The new Act serves to raise the issues and profiles of mental health and to support mental health care users (MHCUs). Some of the objectives of the Act are to: (i) ensure that appropriate care, treatment and rehabilitation (CTR) are provided at all levels of the health service; (ii) change from the custodial approach of the past to one that encourages community care; and (iii) entrench the rights of people with mental disabilities so that they are not discriminated against, stigmatised and/or abused.

The Act goes further to remove distinctions between health professionals, in that any registered medical practitioner or psychiatricallytrained nurse/occupational therapist/psychologist/ social worker (defined as a 'mental health care practitioner') is allowed to provide mental health CTR services. This definition has expanded the base of health care professionals capable of implementing the Act, so that it is not limited to psychiatrists as was the case with the previous Act. The responsibility for clinical decisions has appropriately moved from the judiciary to mental health care practitioners. In the previous Act it was the responsibility of the judicial services (magistrates) to make a decision, based on reports by clinicians and representations by families, with regard to the need for admitting MHCUs without consent. The Act also provides for a 72-hour assessment before further involuntary CTR. It is during these first 72 hours that patients may be most aggressive and need to have some form of restraint imposed on them. Treatment during this 72 -hour period may result in an improvement in the user's capacity to consent and/or a 
reduction in the level of dangerousness to self and others, to the point that the user may voluntarily consent to further treatment or be able to be discharged.

Finally, Mental Health Review Boards (MHRBs) have been established in accordance with the requirements of the Act and a number of international and regional human rights treaties to which South Africa is a signatory. Guided by the provisions of the Constitution, MHRBs must adhere to the values espoused in these human rights instruments and assist in promoting and protecting the human rights of people with mental disorder and intellectual disability. Clearly MHRBs are not the only bodies that have an important role in protecting human rights, nor does their role in terms of the Act cover all aspects of the human rights of people with mental illness and intellectual disability. Nonetheless, MHRBs have an essential and legally specified role. Their administrative powers and functions relating to the CTR of MHCUs are summarised in section 19 of the Act. '

It is recognised internationally that the majority of MHCUs should be treated as voluntary patients. However, a minority will require admission and treatment without their consent. It has been estimated that worldwide 10 - 15\% of MHCUs require involuntary admission. ${ }^{2,3}$ However, the percentage differs considerably between countries because of factors such as lack of services, poor capacity to manage patients, and inadequate early detention and management of problems. Despite these differences, most people and countries seem to agree that the assessment of mental illness and intellectual disability and the decision to treat without consent fall within the medical domain, but that the legal step of restricting a person's freedoms and treating without consent needs to be made by a judicial or quasi-judicial body. ${ }^{4}$ Gable et al. ${ }^{3}$ state that 'Substantive due process requires a determination that involuntary admission is based on accepted psychiatric criteria le.g. existence of a recognized mental illness, threat of imminent harm or deterioration, or necessity of institutional treatment). Procedural due process requires that legal procedures for involuntary admission are followed, that these decisions are made by qualified professionals and not arbitrarily.'

With regard to the above, section 33 of the $\mathrm{MHCA}^{\prime}$ allows that a MHCU must be provided with CTR services without his or her consent (involuntarily) if at the time of making the application there is reasonable belief that the MHCU has a mental illness of such a nature: (i) that the user is likely to inflict serious harm on him- or herself or others; (ii) that the financial interests or reputation of the user need to be protected; (iii) that the user is incapable of making an informed decision on the need for the CTR; and (iv) that he or she is unwilling to receive the CTR required. The Act has a unique category called 'assisted care, treatment and rehabilitation'. Section $26^{\prime}$ states that an MHCU may be provided with assisted CTR without his or her consent (involuntarily) if at the time of making the application there is a reasonable belief that the $\mathrm{MHCU}$ is suffering from a mental illness or severe or profound mental disability of such a nature that he or she is incapable of making an informed decision on the need for the CTR. In both these categories, the decision to treat an MHCU without consent is made by the clinicians and it is the function of the MHRB to consider all such applications to ensure that due process is followed and the rights of the user are upheld in approving or rejecting this decision. The MHRB then submits these applications to the High Court judge for consideration and ratification. MHRBs also receive periodic reports on the mental health status of all MHCUs undergoing prolonged assisted and involuntary CTR.

Gauteng is one of nine provinces in South Africa, with an estimated population of approximately 10 million people. ${ }^{6}$ Cross-border flow from neighbouring provinces and countries increases this figure by several more millions. Because of the high prevalence of mental illness, there are large numbers of MHCUs in this province. Two MHRBs have been in operation since implementation of the MHCA, dealing with applications from the various inpatient facilities, community psychiatric outpatient clinics and long-term care and rehabilitation centres in the northern and southern parts of the province. An adequate information system is therefore needed to capture all applications, track the movement of users within health establishments in the province, and ensure that users receive appropriate CTR at the various service levels. This information management is the responsibility of the board's secretariat.

The MHCA was implemented just over 5 years ago, and it is vital that its relevance, effectiveness and procedures be analysed. While the Act and the regulations are clear in their requirements, the level of implementation thereof still needs to be determined. This can be done by ongoing audit and by academic enquiry. The former involves collating the statistics pertaining to the use of the Act and the latter investigation of the impact the Act has had on mental health services (usually by comparison with other sets of data), thus providing insights into how the legislation or clinical practices (and services) could be modified. The aim of this preliminary study was to investigate the Act's impact by means of an audit of the number and origin of applications for involuntary admissions of MHCUs made to the MHRBs by the various institutions in Gauteng in 2008. It is hoped that this will aid in the design of a further academic study. 


\section{Methods}

This was a retrospective review of the register/database of the two MHRBs in Gauteng. All documents relating to any application made in accordance with the MHCA were submitted to the MHRB offices located at the Gauteng Department of Health in Saver Street, Johannesburg. The secretariat of the board records all incoming documents in the register/database. Files are prepared for consideration by MHRB members, and the decision made by the MHRB is also recorded in the register/database.

The study population consisted of all MHCUs for whom applications for CTR without consent were made to the MHRBs in accordance with the MHCA during the period January - December 2008. There were no exclusion criteria. The investigator accessed the database and extracted the following information for each MHRB: the number of applications per institution and per region for involuntary and assisted inpatient admissions; involuntary or assisted outpatient CTR; and prolonged involuntary or assisted inpatient and/or outpatient CTR. In addition, the total number of admissions per institution with dedicated psychiatric services was obtained from: (i) specialised psychiatric hospitals - Sterkfontein, Weskoppies, Tara; (ii) tertiary academic hospitals - Chris Hani Baragwanath, Dr George Mukhari, Charlotte Maxeke Johannesburg Academic; and (iii) regional hospitals -
Kopanong, Helen Joseph Rahima Moosa, Leratong, Natalspruit and Tembisa.

The study was approved by the University of the Witwatersrand's Human Research Ethics Committee. All personal details of MHCUs were kept confidential.

\section{Results}

\section{Applications for inpatient care}

During the 1-year study period the two MHRBs received a total of 3803 applications for inpatient care, of which $66.4 \%$ ( $N=2526$ ) were for assisted inpatient care and $33.6 \%(N=1277)$ for involuntary inpatient care (Table I); $48.1 \%(N=1831)$ of these applications were from the regional hospitals, $29.6 \%(N=1127)$ from specialised psychiatric hospitals and 22.2\% (N=845) from tertiary academic hospitals.

Of the applications for involuntary inpatient CTR, 73.1\% were from the specialised psychiatric hospitals, the majority (65.2\%) being from Sterkfontein Hospital. Of the admissions for assisted inpatient CTR, 70.3\% were from the regional hospitals (Kopanong 18.7\%, Leratong 13.3\%). Of note was that neither of these hospitals submitted any applications for involuntary inpatient care. Similarly, Charlotte Maxeke Johannesburg Hospital in the

Table I. Frequency distribution of application for inpatient care (involuntary and assisted)

\begin{tabular}{|c|c|c|c|c|c|c|c|}
\hline \multirow[b]{2}{*}{ Hospital } & \multirow{2}{*}{$\begin{array}{l}\text { No. of } \\
\text { inpatient beds }\end{array}$} & \multicolumn{2}{|c|}{$\begin{array}{l}\text { All MHCA admissions } \\
\qquad(N=3803)\end{array}$} & \multicolumn{2}{|c|}{$\begin{array}{l}\text { Assisted } \\
(N=2526)\end{array}$} & \multicolumn{2}{|c|}{$\begin{array}{l}\text { Involuntary } \\
(N=1277)\end{array}$} \\
\hline & & $N$ & $\%$ & $N$ & $\%$ & $N$ & $\%$ \\
\hline Specialised & 1151 & 1127 & 29.6 & 194 & 7.7 & 933 & 73.1 \\
\hline Sterkfontein & 283 & 833 & 21.9 & 0 & 0.0 & 833 & 65.2 \\
\hline Tara & 141 & 71 & 1.9 & 66 & 2.6 & 5 & 0.4 \\
\hline Weskoppies & 727 & 223 & 5.9 & 128 & 5.1 & 95 & 7.4 \\
\hline Tertiary academic & 223 & 845 & 22.2 & 555 & 22.0 & 290 & 22.7 \\
\hline Chris Hani Baragwanath & 155 & 168 & 7.0 & 251 & 9.9 & 17 & 1.3 \\
\hline C M Johannesburg & 20 & 19 & 0.5 & 19 & 0.8 & 0 & 0.0 \\
\hline Dr George Mukhari & 48 & 558 & 14.7 & 285 & 11.3 & 273 & 21.4 \\
\hline Regional & 142 & 1831 & 48.1 & 1777 & 70.3 & 54 & 4.2 \\
\hline Helen Joseph & 32 & 54 & 1.4 & 43 & 1.7 & 11 & 0.9 \\
\hline Kopanong & 30 & 712 & 18.7 & 712 & 28.2 & 0 & 0.0 \\
\hline Leratong & 32 & 504 & 13.3 & 504 & 20.0 & 0 & 0.0 \\
\hline Natalspruit & 18 & 193 & 5.1 & 163 & 6.5 & 30 & 2.3 \\
\hline Tembisa & 30 & 368 & 9.7 & 355 & 14.1 & 13 & 1.0 \\
\hline
\end{tabular}


tertiary academic group also had no applications for involuntary inpatient care.

\section{Applications for outpatient care}

During the study period 1226 applications were received by the MHRBs for outpatient CTR, of which the majority lapproximately 92\%) were for assisted outpatient CTR (Fig. 1).

The health establishments in northern Gauteng accounted for $71.7 \%$ of these applications (assisted 66.3\%, involuntary 5.4\%), while those in southern Gauteng accounted for $28.3 \%$ lassisted $25.3 \%$, involuntary $3.0 \%$ ). Although the health establishments in northern Gauteng applied for more outpatient CTR compared with those in southern Gauteng (879 v. 347, respectively), the ratios of assisted to involuntary outpatient applications for CTR within each region were similar lapproximately 12:1 and 9:1, respectively).

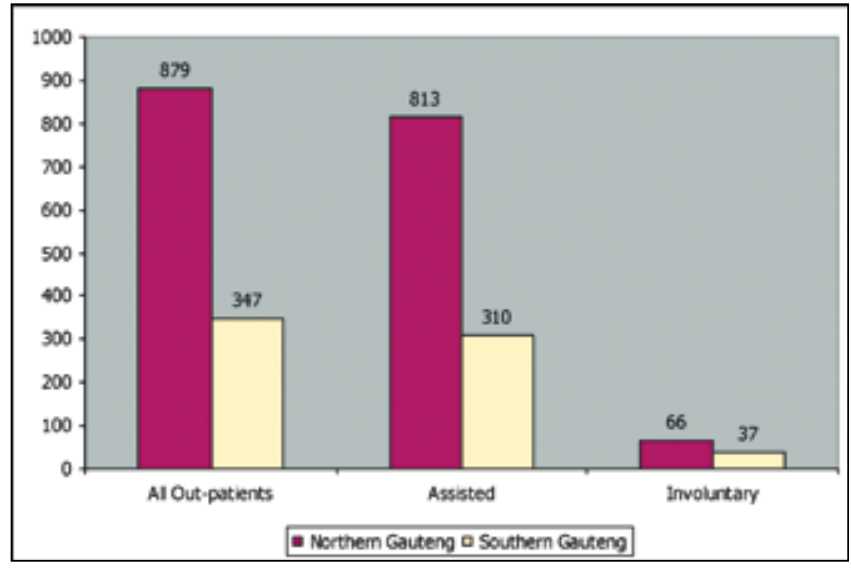

Fig. 1. Frequency distribution of applications for outpatient care (involuntary and assisted).

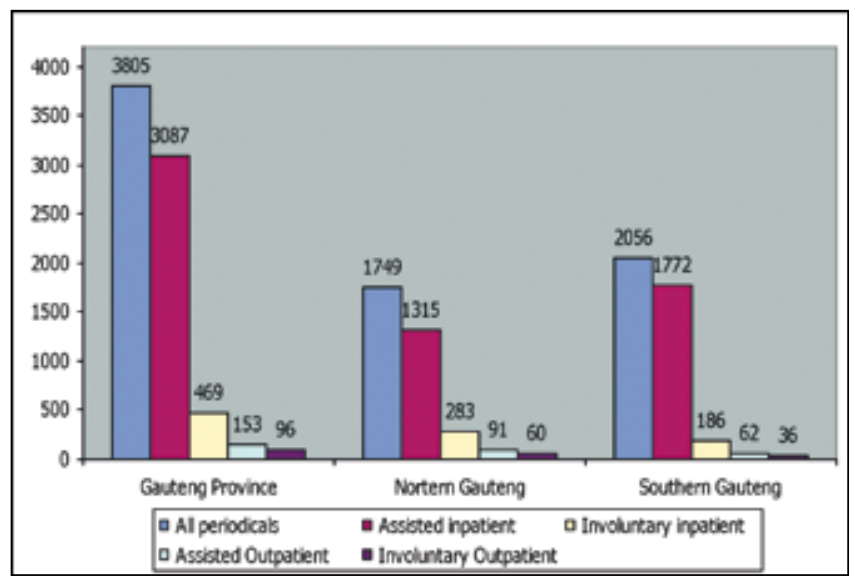

Fig. 2. Frequency distribution of periodical reports received by the MHRBs.

\section{Applications for prolonged inpatient or outpatient care}

The boards received 3805 periodic reports for prolonged CTR, of which $93.5 \%$ ( $N=3$ 556) were for inpatient and $6.5 \%(N=249)$ for outpatient CTR (Fig. 2). The majority of the applications for continued inpatient (86.8\%) and outpatient CTR (61.5\%) were for assisted CTR.

\section{Discussion}

\section{Involuntary admissions}

In psychiatric practice worldwide, review of legislation providing the framework for mental health care coupled with persistent lobbying from the human rights movements has resulted in a shift in the basic criteria for providing mental health care. The paternalistic approach by the service provider is being replaced by an approach that involves the patient and family and entrenches the rights of the mentally ill. A major objective is to reduce admission and treatment without consent. The rate of involuntary admission and care is therefore widely considered to be an indicator of the level of implementation of these reforms.

The annual number of involuntary admissions per 100000 population differs in various countries: 24.4 in Denmark, 24.8 in Sweden, ${ }^{7} 43.5$ in Greenland, ${ }^{8} 9.4$ - 108.8 in the sixteen Federal States of Germany, 11 in France, and 48 in the UK. ${ }^{9,10}$ This study found the annual rate in Gauteng to be 12.8/100 000. Although this figure lies within the wide range given above, it is lower than that of most developed countries. It is possible that under the South African MHCA, the exclusion of assisted admissions /also subject to admission and treatment without consent) may contribute to these lower figures. If assisted admissions are included the frequency increases threefold to 38.3/100000 population.

Further, in sharp contrast to the intentions of mental health reforms, slowly increasing annual rates of involuntary admission have been reported in most countries, ${ }^{11,12}$ except Sweden, where it decreased from 116 in 1979 to 19.7 in 1993.13 Our Gauteng study also showed an increase in the annual rate of involuntary admissions from 6.6/100 000 in 2007 lunpublished data from the MHRB database) to 12.8 in 2008 . This almost doubling in involuntary admissions could reflect a disregard by Gauteng health professionals of the principle of ensuring that users are treated without their consent only when absolutely necessary, as is stated in the new mental health reforms. Alternatively, stakeholders (service providers, administrators and MHRB) may have gained 
experience and a better understanding of the Act, resulting in general improved levels of implementation and reporting of all the various forms of admissions, and consequently more involuntary admissions. This latter view is supported by the huge drive of the Gauteng Mental Health Directorate and the MHRBs to conduct training on implementation of the Act at all institutions in Gauteng.

Although not supported by this study, some service providers suggest that more patients are presenting with psychiatric disorders that necessitate involuntary admissions, e.g. a higher proportion of psychiatric patients are misusing drugs and alcohol, leading to more florid presentations of psychotic illness, and delays in admission and treatment caused by bed shortages may mean that patients' illnesses are more severe at presentation. ${ }^{14}$ Further study into the reasons for involuntary admissions is therefore warranted.

We also found that the specialised hospitals as a group had more applications for involuntary admissions than the regional hospitals. According to section 34(4) of the MHCA, before involuntary admission an MHCU should undergo a '72 hour assessment' during which time the user is investigated and treated without consent. At the end of that period a decision should be taken with regard to the need for involuntary admission. If the $\mathrm{MHCU}$ is to be provided CTR on an inpatient basis, he or she must be transferred to a psychiatric hospital. Treatment of involuntary patients requires, among other things, an infrastructure designed for this service, 'psychiatric intensive care' capabilities, special staffing, training and supervision, ancillary services and funding. Such services are lacking at most regional hospitals in Gauteng, so the 72-hour assessments are conducted at regional hospitals and the $\mathrm{MHCU}$ is then referred to a specialised psychiatric hospital for further involuntary inpatient CTR. This accounts for the higher number of applications by specialised psychiatric hospitals.

It is possible that the number of involuntary admissions at regional hospitals will increase in the future because Gauteng, like other densely populated large cities, tends to have relatively high rates of involuntary admissions ${ }^{15}$ and the number of beds available at specialised psychiatric hospitals is limited. It is recommended that the Department of Health plans for this eventuality by improving both the infrastructure and human resources in regional hospitals. In the interim, the willingness of a regional hospital to admit involuntary patients should be contingent on its being able to control its admissions, not being the provider of last resort, and receiving support from the specialised psychiatric hospitals.

\section{Further involuntary CTR}

\section{Outpatient care}

The MHCA requires that if further care is provided without the patient's consent, it should be done in the least restrictive environment and as close to the patient's home as possible. When the patient's condition improves to a point at which they are considered not to be a danger to themselves or others, but still lack the capacity to consent to voluntary treatment, they should be discharged into the care of their families and be managed as involuntary outpatients at a local community psychiatric clinic. The intention of the Act is to empower the family and help ensure that they play an active role in the ongoing treatment of the MHCU. It is also intended to improve treatment adherence and prevent relapse and the 'revolving door' syndrome.

During the study period there were significant numbers of applications for involuntary outpatient CTR from both regions of Gauteng, indicating a positive trend by mental health care providers (albeit more in northern Gauteng) to implement this regulation of the MHCA. Further research is needed to determine the success of this involuntary outpatient approach.

\section{Long-term involuntary care}

The MHCA requires that treatment without consent be provided for as short a time as possible. If it has to be provided for a continued period of more than 6 months, it must be reviewed regularly and periodic reports must be submitted for approval to the MHRBs, justifying the continued care and the lack of capacity to consent. During the study period the MHRBs received 3805 such reports. Although this represents a significant number of users, the majority of these applications were for the long-term/prolonged assisted inpatient CTR of intellectually disabled or severely cognitively impaired users in non-governmental residential homes and privately contracted institutions. The capacity of intellectually disabled or severely cognitively impaired users to provide informed consent is very unlikely to improve because of the nature of their illness. The MHCA aims that these users should be transferred from state hospitals to be cared for in non-governmental residential homes within their community. There were, however, a significant number of applications for prolonged involuntary CTR from specialised psychiatric hospitals despite a long admission within the facility. These users remain a danger to themselves and/or others, lack the capacity to make an informed decision, and refuse treatment. Studies have recommended that prolonged involuntary hospitalisation of such patients must continue despite the risk of institutionalisation. ${ }^{16}$ These patients would not cope in 
the community and would accumulate in acute inpatient hospital units, revolve in and out of acute inpatient units, be homeless or be poorly cared for. ${ }^{17}$ Trieman and Leff ${ }^{18}$ state that such patients can only be discharged from specialised psychiatric hospitals if specialised long-stay facilities are available.

Because this is a retrospective study of information captured on the database of the secretariat of the MHRBs, it is likely that some data were missing and perhaps incorrectly captured. The lack of socio-demographic and psychopathological details for involuntary admissions prevents further analysis and is an important limitation of this study. Although, the diagnoses would have been helpful to determine the reason for admission (psychotic illnesses, substance-induced disorders, mood disorders, behavioural disturbances, etc.l, often the diagnoses on the application forms are provisional and made by generalist physicians. These vary considerably from the final diagnoses made by specialist psychiatrists, which call their reliability into question. Finally, this study is not inclusive of all the factors that are associated with implementation of the Act. However, it did focus on the important ones, which have clear procedures and impact on users' rights, and it gives an assessment of the level of implementation of the MHCA in Gauteng, albeit a preliminary one.

An interesting and important follow-up study would be to investigate whether the MHRBs are complying with the Act themselves, whether they submit their forms to the High Court judges correctly, and whether the judges agree with their process.

\section{Conclusion}

The MHCA and its regulations support the appropriate care of mentally ill persons without depriving them of their rights. Although the procedures in the Act are clear, the legal, political, economic, social, medical, methodological and other factors prevailing in Gauteng province and interacting in the process of care of people with mental illness are complex and still poorly understood, and impact on the level of implementation of the Act. Further, it would appear that in the 4 years since the Act's promulgation there have been significant strides towards implementation of the procedures relating to involuntary admission and CTR of MHCUs by all stakeholders. Differences in levels of knowledge of the various stakeholders may result from differences in perceptions, attitudes and understanding of their roles and therefore indicate a massive need for education of mental health care professionals and the public. The Department of Health needs to invest more funds to improve mental health human resources and infrastructure at all health establishments.

\section{References}

1. Mental Health Care Act, No. 17, 2002. Pretoria: Department of Health, 2004 http://www.info.gov.za/gazette/acts/2002 (accessed 29 December 2008).

2. Salize HJ, Drebing H, Peitz M. Compulsory Admission and Involuntary Treatment of Mentally III Patients in Legislation and Practice. Mannheim, Germany: EU Member States, 2002

3. Gable L, Vasquez J, Gostin L, Jimenez H. Mental health and due process in the Americas: protecting the human rights of persons involuntarily admitted to and detained in psychiatric institutions. Pan Am J Public Health 2005; 1 8:366-373.

4. World Health Organization. WHO Resource Book on Mental Health, Human Rights and Legislation. Geneva: WHO, 2005. www.who.int/mental_health/.../who_rb_ mnh_hr_leg_FINAL_11_07_05.pdf (accessed 14 March 2009).

5. The Mental Health Care Act, No. 17, 2002: Regulations; Chapter IX, Section 66: 1 (k). Pretoria: Department of Health, 2004. http://www.info.gov.za/gazette/ acts/2002 (accessed 29 December 2008).

6. South Africa: Population Census 2001. Community profile database. Pretoria: Statistics SA. hitp: // www.statssa.gov.za/census $01 / \mathrm{html} /$ default.asp laccessed 14 March 2009).

7. Riecher-Rössler A, Rössler W. Compulsory admission of psychiatric patients - an international comparison. Acta Psychiatr Scand 1993;87:23 1-236.

8. Engberg M. Involuntary commitment in Greenland, the Faeroe Islands and Denmark. Acta Psychiatr Scand 1991;84:353-356.

9. Salize HJ, Dressing H. Epidemiology of involuntary placement of mentally ill people across the European Union. Br J Psychiatry 2004; 1 84:163-168.

10. Laffont I, Priest RG. A comparison of French and British mental health legislation. Psychol Med 1992;22:843-850

11. Wall S, Hotopf M, Wessely S, Churchill R. Trends in the use of the Mental Health Act: England 1984-96. BMJ 1999;318:1520-1521

12. Lecompte $D$. The paradoxical increase in involuntary admissions after the revision of the Civil Commitment Law in Belgium. Med Law 1995; 14:53-57.

13. Kjellin L. Compulsory psychiatric care in Sweden 1979-1993. Soc Psychiatry Psychiatr Epidemiol 1997:32:90-96.

14. Cuffel B. Prevalence estimates of substance abuse in schizophrenia and their correlates. J Nerv Ment Dis 1992; 180:589-592.

15. Malla A, Norman RMG. Involuntary admissions in a Canadian province: the influence of geographic and population factors. Soc Psychiatry Psychiatr Epidemiol 1988;23:247-251.

16. Mackay RD, Ward T. The long-term detention of those found unfit to plead and legally insane. British Journal of Criminology 1994;34:30-43.

17. Shepherd G. System failure? The problems of reductions in long-stay beds in the UK Epidemiology Psychiatry and Society 1998;7:127-134

18. Trieman N, Leff J. The difficult to place patients in a psychiatric hospital closure programme. The TAPS project 24. Psychol Med 1996;26:765-774.

19. Bradeley C, Marshall M, Gath D. Why do so few patients appeal against detention under section 2 of the mental health act? BMJ 1995:310:364-367. 